\title{
Determinants of Femoral Tunnel Length in Anterior Cruciate Ligament Reconstruction: CT Analysis of the Influence of Tunnel Orientation on the Length
}

\author{
Geethan Iyyampillai, MS, Easwar Thirunellai Raman, MS, David Vaithyalingam Rajan, MS, \\ Ajith Krishnamoorthy, MS, and Santhosh Sahanand, MS \\ Department of Orthopaedic Surgery, Ortho One Orthopaedic Speciality Center, Coimbatore, India
}

\begin{abstract}
Purpose: The purpose of the study was to identify the femoral tunnel orientation that consistently results in a tunnel length of more than 35 mm in anterior cruciate ligament reconstruction.

Materials and Methods: Computed tomography (CT) scans were obtained from 30 patients who did not have any pathology in the distal femur. Virtual tunnels were marked on 3D (3-dimensional) reconstructed CT images after determining the orientation defined by sagittal inclination and axial angle. The length of a femoral tunnel in 64 different combinations of orientations (between $30^{\circ}$ and $65^{\circ}$ in $5^{\circ}$ increments in two planes) was measured on $3 \mathrm{D}$ reconstructed images in both knees in 30 patients. Reliability of measurement was assessed with correlation coefficient for intraobserver and inter-observer measurements. A one-way analysis of variance (ANOVA) was used for statistical analysis.

Results: The mean femoral tunnel length varied significantly with each $10^{\circ}$ change in orientation in both planes ( $\mathrm{p}<0.05$, ANOVA). A femoral tunnel of more than $35 \mathrm{~mm}$ in length could be obtained only with a sagittal inclination of more than $50^{\circ}$ and axial angle of $30^{\circ}-45^{\circ}$. When the axial angle was kept constant, the tunnel length increased with the increase in sagittal inclination. Higher sagittal inclinations consistently resulted in longer tunnels $(\mathrm{r}>0.9)$ and reduced the incidence of posterior cortical breakage.

Conclusions: A tunnel orientation with an axial angle between $30^{\circ}-45^{\circ}$ and a sagittal inclination of more than $50^{\circ}$ can result in a tunnel length of more than $35 \mathrm{~mm}$.
\end{abstract}

Keywords: Knee, Anterior cruciate ligament, Reconstruction, Arthroscopy, Imaging, three-dimensional

\section{Introduction}

Anterior cruciate ligament (ACL) reconstruction improves stability and function of the knee and reduces the risk of chondral and meniscal injuries. However, it does not seem to protect the

Received April 10, 2013; Revised (1st) June 22, 2013;

(2nd) August 20, 2013; (3rd) September 26, 2013;

Accepted September 27, 2013

Correspondence to: Geethan Iyyampillai, MS

Department of Orthopaedic Surgery, Ortho One Orthopaedic Speciality Center, 657, Trichy Road, Singanallur, Coimbatore, Tamil Nadu 641-005, India

Tel: +91-988-452-2103, Fax: +91-422-232-1288

E-mail: igeethan@gmail.com

This is an Open Access article distributed under the terms of the Creative Commons Attribution Non-Commercial License (http://creativecommons.org/licenses/by-nc/3.0/) which permits unrestricted non-commercial use, distribution, and reproduction in any medium, provided the original work is properly cited. knee from degenerative arthritic changes in all patients ${ }^{1)}$. Hence, the techniques in ACL reconstruction continue to evolve and many issues are still under debate. Creation of a femoral tunnel by drilling through an anteromedial (AM) portal has been a significant innovation in the recent years ${ }^{2}$. This technique allows independent creation of a femoral tunnel from a tibial tunnel and improves restoration of anatomy ${ }^{3)}$ and stability in ACL reconstruction $^{2,4,5)}$. On the other hand, short tunnel length remains as a disadvantage $e^{6-8)}$. Short tunnels might result in inadequate early graft fixation strength and might cause difficulties in suspensory fixation ${ }^{9)}$. Previous cadaveric and clinical studies reported the mean femoral tunnel length drilled through an AM portal as ranging from $15.7-34.2 \mathrm{~mm}^{6-8)}$. We suspected that this wide variation could be due to different tunnel positions and orientations employed by different authors. We perform anatomic single bundle ACL reconstruction with the limb draped free rather than fixed in a knee leg holder (Fig. 1). In this position, the hip flexion 

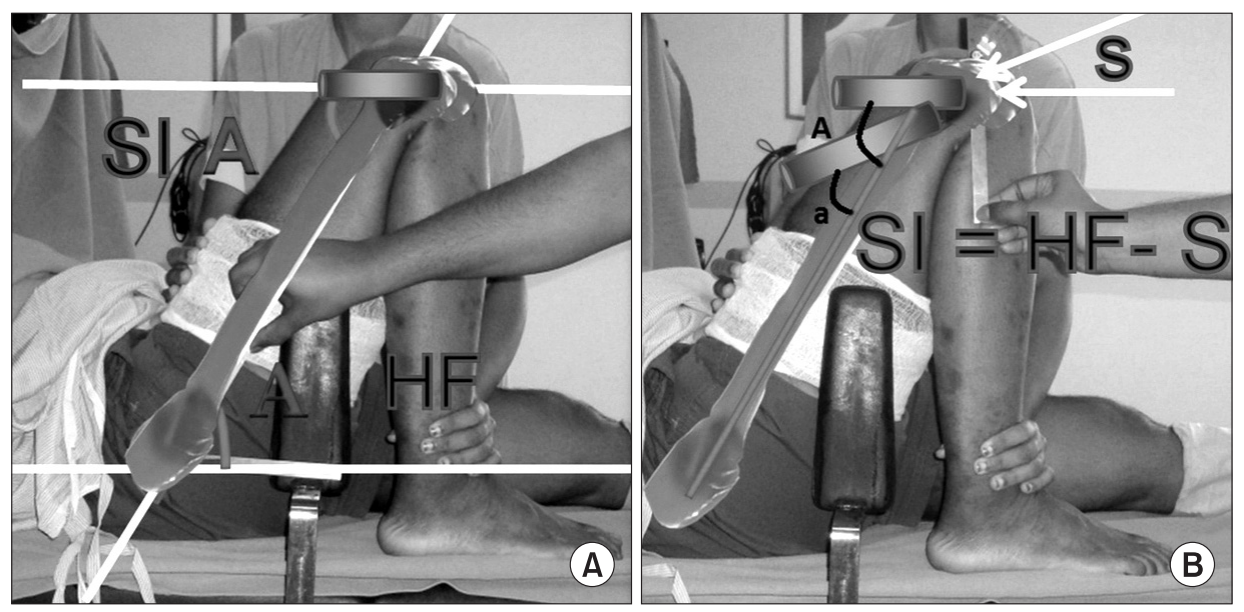

Fig. 1. Determinants of Sagittal Inclination. (A) $\mathrm{SI}=\mathrm{HF}$ when the guidewire is parallel to the ground. (B) SI=HF-S when the guidewire is not parallel to the ground. SI: sagittal inclination, HF: hip flexion, A: angle of hip flexion, a: sagittal angle of guidewire, S: sagittal angle of the guidewire.

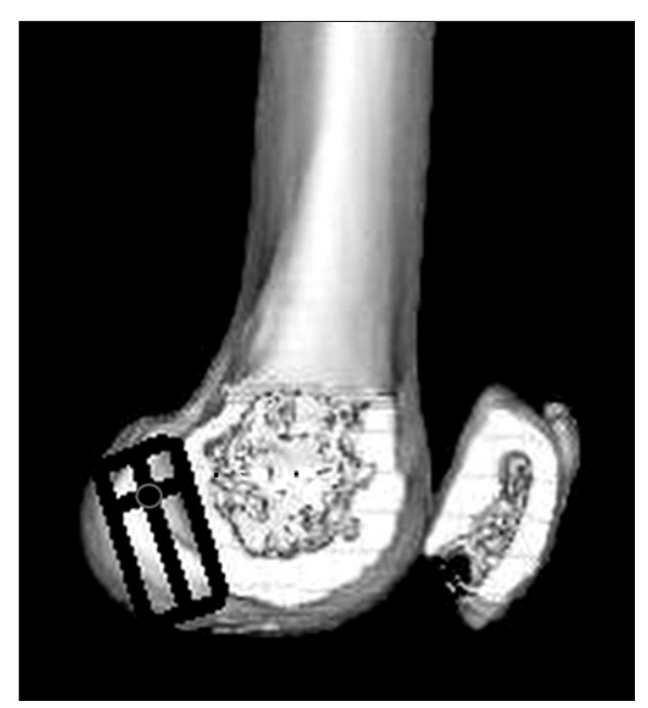

Fig. 2. Femoral tunnel entry point was marked based on the grid method at the midpoint of a perpendicular line drawn from a point at $5 / 6$ th of a line tangent to the roof of the notch.

varies with knee flexion and studies in the available literature do not account for this fact. The available studies on this subject have analysed the tunnel length with respect to knee flexion during femoral tunnel drilling or axial angle of guidewire ${ }^{4,10,11)}$. However, there is no study objectively analysing the three dimensional relationship between tunnel orientation and length. The purpose of this study was to investigate if the tunnel length varies significantly in different orientations and to determine the 3-dimensional (3D) optimal drilling angle of the trans-portal technique for appropriate femoral tunnel length. We hypothesized that tunnel length varies with orientation and there exists a tunnel orientation in which a tunnel of optimal length can be obtained.
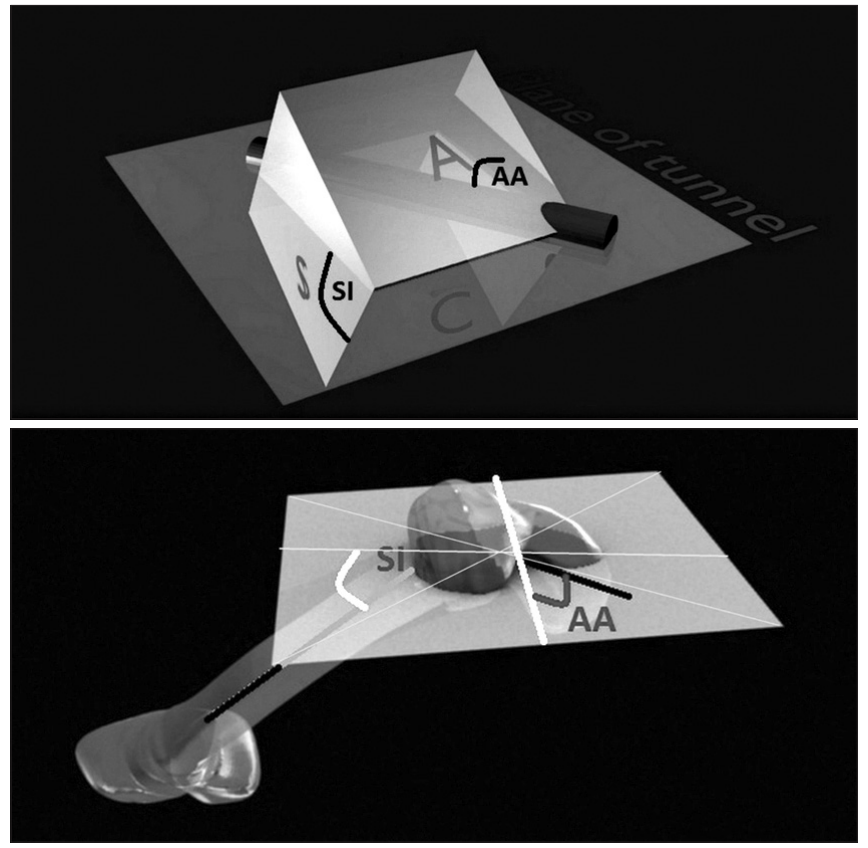

Fig. 3. Sagittal inclination (SI) was measured by projection of the angle in the true sagittal plane (S) on the sagittal plane. Axial angle (AA) was measured as the angle made on the imaginary plane (A) along the tunnel.

\section{Materials and Methods}

We conducted this study using computed tomography (CT) images stored in the hospital database. The inclusion criteria were age between 20 and 40 years, no pathology in the distal femur and no history of prior surgery. Thirty continuous CT records satisfying the inclusion criteria were included in the study. Height and weight of all patients were noted from hospital records. The study sample had 25 males and five females. Age ranged from 23 to 38 years, (Mean 27 years). The mean height was $174 \mathrm{~cm}$ (range, 155 
to $182 \mathrm{~cm}$ ). Though CT was indicated for only one knee, the CT image acquisition protocol of our hospital included both knees. For the purpose of this study, we did 3D CT reconstruction from the available database of both knees (Sample size: 30 patients, 60 knees). The CTs were acquired with Seimens Somatom scanner and images were analysed with Synco Software (Seimens Heathcare, Malvern, PA, USA). An entry point was marked on the 3D reconstructed images as reported in the previous studies on single bundle anatomic reconstruction $^{12}$. The medial condyle was subtracted from the image and the end face view of the lateral femoral condyle was obtained. A line was drawn along the roof of the notch. A point five sixths of the line from distal to proximal was chosen. A perpendicular line was drawn from this point towards the chondral margin. The midpoint of this line was chosen as the entry point (Fig. 2). Orientation of the femoral tunnel position in a $3 \mathrm{D}$ plane is not easily measured per-operatively. Hence, we resolved this problem by defining the tunnel orientation in a sagittal plane and an oblique axial plane (Fig. 3). Angles in these two planes, the sagittal inclination in the sagittal plane and axial angle in the oblique axial plane, could be measured intra-operatively and were used to define tunnel position on the reformatted CT images. We noted that the tunnel orientation along the sagittal plane (sagittal inclination) varies with the degree of hip flexion (Fig. 1) and the sagittal angle of guidewire insertion (Fig. 4). We obtained oblique axial cuts along the various sagittal inclination planes in $5^{\circ}$ increments (Fig. 5) and virtual tunnels were created along the various axial angles (Fig. 6) and the tunnel length was measured. The measurements were made in 64 different orientations, between $30^{\circ}$ and $65^{\circ}$, in $5^{\circ}$ increments, in both sagittal and oblique axial planes. All measurements were performed on a picture archiving and communication system monitor (Synco Software; Seimens Heathcare) by use of a mouse-point cursor

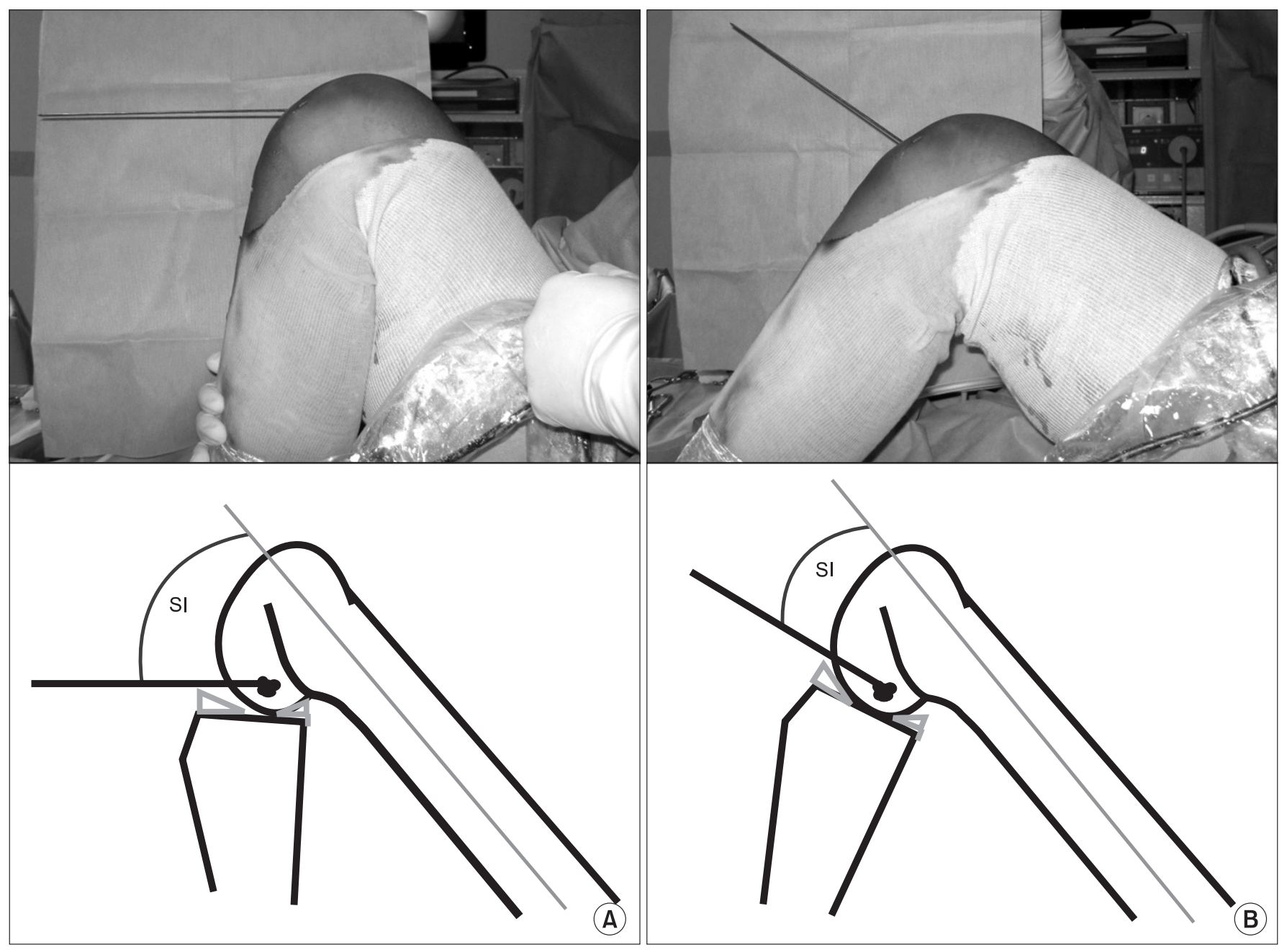

Fig. 4. Influence of knee flexion on the sagittal inclination. (A) Guidewire is parallel to ground when knee is fully flexed. (B) Guidewire is not parallel to ground when knee is not fully flexed. SI: sagittal inclination. 

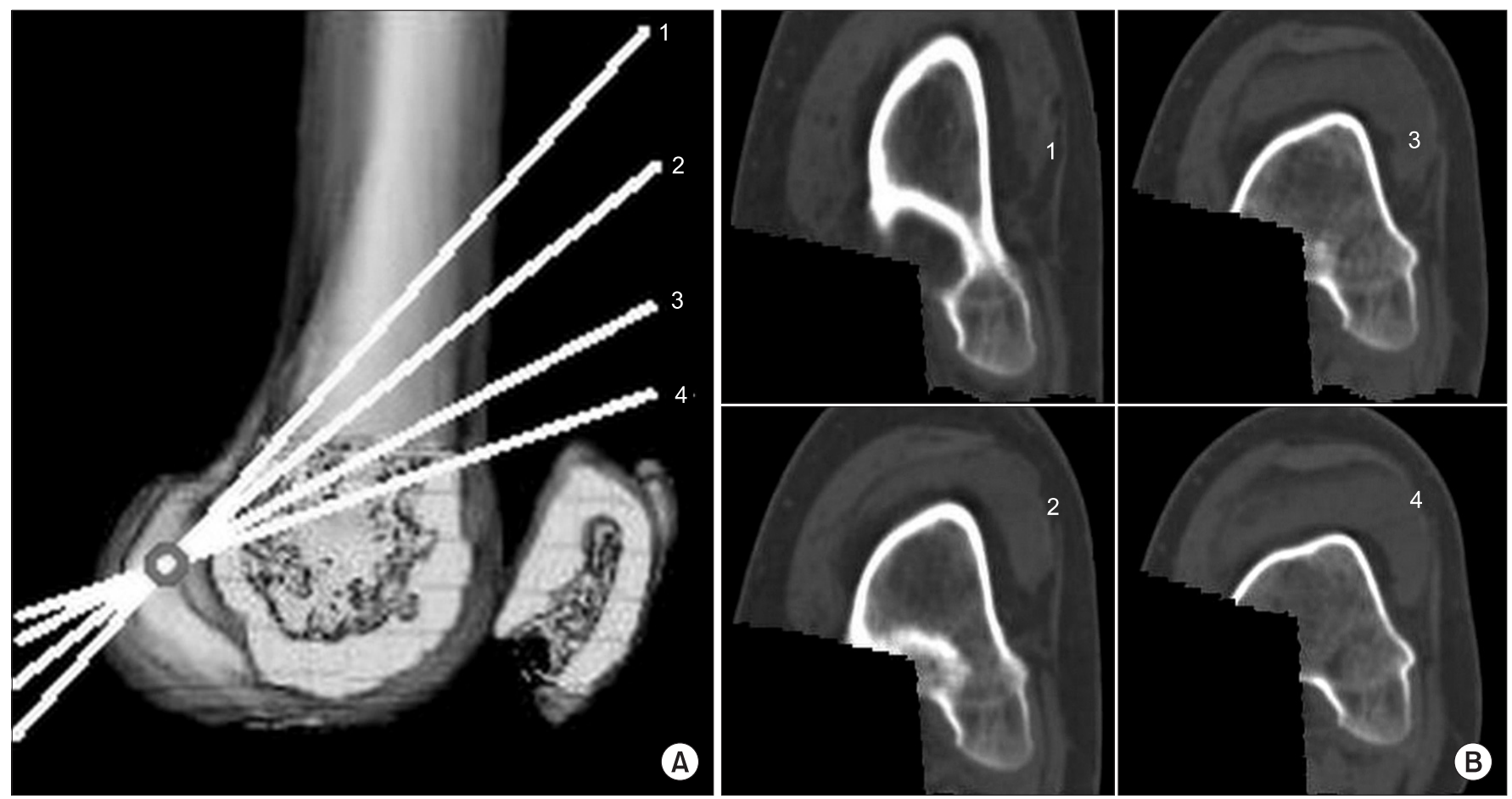

Fig. 5. (A) Lines 1-4 depict various sagittal inclinations along which the cross sectional images of distal femur are reconstructed. (B) Cross sectional computed tomography images obtained along the respective sagittal inclinations.
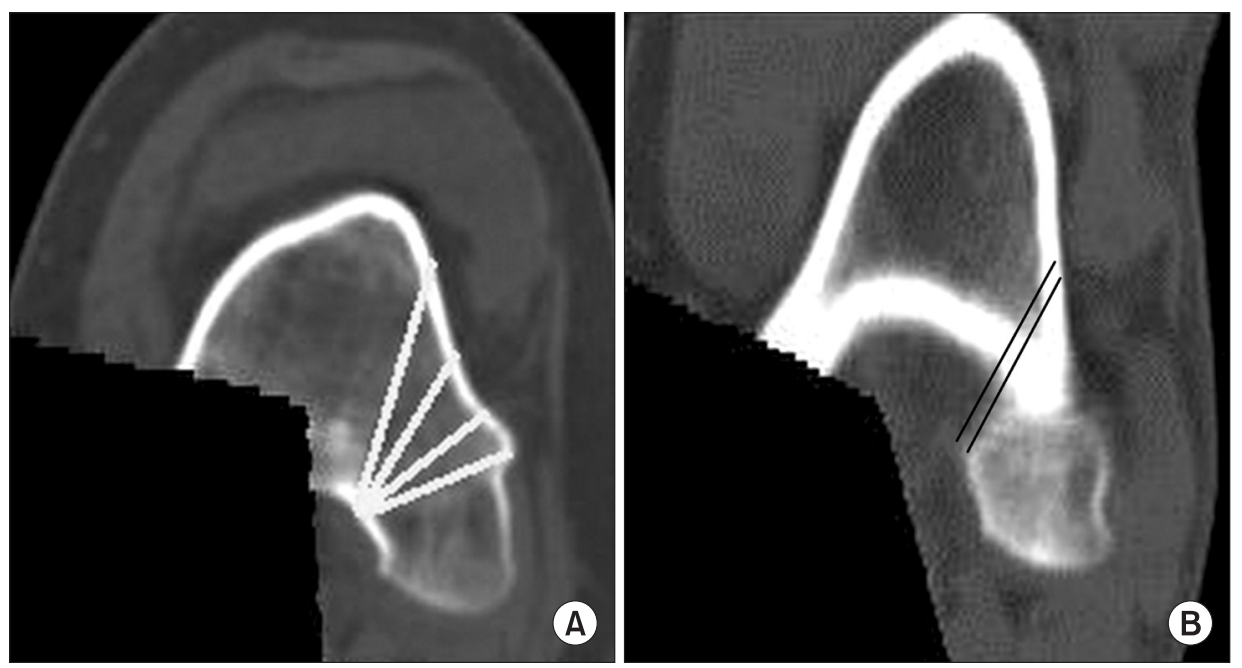

Fig. 6. Virtual tunnels created along various axial angles. (A) Posterior cortex intact along all axial angles. (B) Posterior cortex is deficient.

and automated computer calculation for distance and angle. Accepting an error of $0.1 \mathrm{~mm}$ for the groove depth and $10^{\circ}$ for the angles, the formula derived from Gaussian distribution ( $\mathrm{n}=[\mathrm{zs} /$ e]2, where $z=1.96$ for $5 \%$ level of significance, $s$ is standard deviation of the sample and e is the accepted error) ${ }^{13)}$, was used and the sample was found to be sufficient to draw conclusions among general population. Inter-observer variation was determined by having two authors independently calculate the tunnel length on $10 \mathrm{CT}$ images that were randomly selected. Intra-observer variability was estimated by having one of the authors calculate the confidence interval of the same radiographs with an interval of 6 weeks. Both were instructed on the use of the software by a musculoskeletal radiologist at the beginning of the study. The measurements were taken independently and sequentially. We used the Pearson correlation coefficient, with scores between 0.61 and 0.80 representing good correlation and those greater than 0.81 representing excellent correlation. We thus plotted an eight by eight table showing tunnel length in each orientation for all 60 
Table 1. Tunnel Length Measured in Various Tunnel Orientations

\begin{tabular}{|c|c|c|c|c|c|c|c|c|}
\hline \multirow{2}{*}{$\begin{array}{c}\text { Sagittal } \\
\text { inclination }\left({ }^{\circ}\right)\end{array}$} & \multicolumn{8}{|c|}{ Axial angle $\left({ }^{\circ}\right)$} \\
\hline & 30 & 35 & 40 & 45 & 50 & 55 & 60 & 65 \\
\hline 30 & $43.12 \pm 2.45$ & $39.11 \pm 1.36$ & $35.87 \pm 2.65$ & $34.75 \pm 2.53$ & $32.25 \pm 2.95$ & $31.12 \pm 2.02$ & $29.87 \pm 1.63$ & $30.51 \pm 1.58$ \\
\hline 35 & $43.32 \pm 2.25$ & $39.33 \pm 2.54$ & $36.43 \pm 2.85$ & $34.1 \pm 1.59$ & $32.77 \pm 3.15$ & $31.3 \pm 2.95$ & $30.8 \pm 2.96$ & $31.79 \pm 2.38$ \\
\hline 40 & $41.9 \pm 2.65$ & $38 \pm 3.19$ & $35.27 \pm 2.76$ & $33.72 \pm 1.81$ & $31.81 \pm 2.62$ & $31.73 \pm 2.65$ & $31.9 \pm 2.56$ & $32 \pm 2.43$ \\
\hline 45 & $41 \pm 3.16$ & $37.81 \pm 2.91$ & $35.36 \pm 2.68$ & $33.9 \pm 2.91$ & $32.54 \pm 2.64$ & $32 \pm 2.43$ & $32.19 \pm 2.58$ & $32.46 \pm 2.16$ \\
\hline 50 & $42.63 \pm 2.51$ & $38.9 \pm 3.12$ & $36.81 \pm 3.65$ & $35.36 \pm 2.16$ & $33.63 \pm 2.59$ & $33.19 \pm 2.49$ & $33 \pm 2.19$ & $33 \pm 2.46$ \\
\hline 55 & $42.54 \pm 2.46$ & $39.63 \pm 2.62$ & $37.54 \pm 2.89$ & $35.72 \pm 2.56$ & $34.37 \pm 2.64$ & $34 \pm 2.73$ & $33.64 \pm 2.53$ & $33.73 \pm 2.58$ \\
\hline 60 & $42.9 \pm 2.32$ & $40.36 \pm 2.61$ & $37.72 \pm 2.36$ & $36.09 \pm 2.68$ & $35 \pm 2.91$ & $33.9 \pm 2.46$ & $33.82 \pm 2.69$ & $33.73 \pm 2.86$ \\
\hline 65 & $43.54 \pm 3.15$ & $40.44 \pm 2.59$ & $38.34 \pm 2.62$ & $36.91 \pm 2.59$ & $35.73 \pm 2.06$ & $34.92 \pm 1.46$ & $34.72 \pm 1.59$ & $34.28 \pm 1.62$ \\
\hline
\end{tabular}

Values are presented as mean \pm standard deviation.

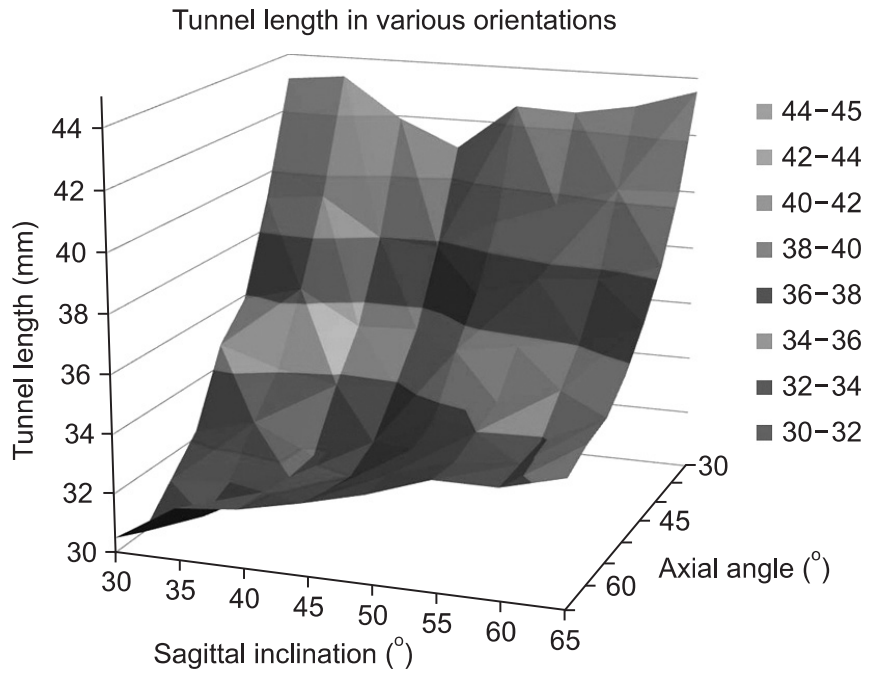

Fig. 7. Femoral tunnel lengths in different orientations.

knees and analysed the mean tunnel lengths in 64 orientations in those 60 knees. A one-way analysis of variance was used to identify the significance of differences between the tunnel lengths in various orientations and the simple linear co-efficient of correlation was calculated to derive the correlation between the tunnel length and orientation. Posterior cortical breakage was identified when the virtual tunnel was not covered by cortical bone (Fig. 6).

\section{Results}

The inter-observer and intra-observer reliability was excellent (Pearson correlation coefficient $[\mathrm{r}]=0.94$ for inter- observer reliability and $\mathrm{r}=0.98$ for intra-observer reliability). The mean lengths of femoral tunnels in the possible orientations are summarised in Table 1 (Fig. 7). We noted that higher sagittal inclina-
Table 2. Correlation Coefficients with Various Axial Angles and Sagittal Inclinations

\begin{tabular}{ccc}
\hline \multirow{2}{*}{ Parameter $\left({ }^{\circ}\right)$} & \multicolumn{2}{c}{ Correlation coefficient } \\
\cline { 2 - 3 } & Axial angle & Sagittal inclination \\
\hline 30 & 0.154 & -0.948 \\
35 & 0.616 & -0.922 \\
40 & 0.821 & -0.884 \\
45 & 0.829 & -0.893 \\
50 & 0.917 & -0.918 \\
55 & 0.976 & -0.926 \\
60 & 0.988 & -0.938 \\
65 & 0.978 & -0.941 \\
\hline
\end{tabular}

tions resulted in longer tunnels but the difference was significant only when the axial angle of the tunnel was more than $40^{\circ}$ (simple linear correlation, $\mathrm{r}<0.6$ for axial angles less than $35^{\circ}$ and $\mathrm{r}>0.9$ for larger axial angles, $r$ - simple linear correlation coefficient, t-test) (Table 2). The tunnel length was observed to decrease with increasing axial angle, and though the results were statistically significant in all sagittal inclinations ( $r>0.85$ for all sagittal inclinations) the correlation was more pronounced at low sagittal inclinations. Tunnel length was more consistent at high sagittal inclinations compared to low sagittal inclinations (coefficient of variance $[\mathrm{CV}]=1.6$ for $30^{\circ}$ sagittal inclination and $\mathrm{CV}=1.03$ for $65^{\circ}$ sagittal inclination). The tunnel length was more than $35 \mathrm{~mm}$ in 57 out of 60 knees (95\%) in all tunnel orientations between axial $30^{\circ}-45^{\circ}$ angles and sagittal $50^{\circ}-65^{\circ}$ inclinations and always more than $30 \mathrm{~mm}$ in these orientations. Outside this range, posterior cortical breakage was noted only in sagittal inclinations of $35^{\circ}$ or less. In this subgroup, axial angles of $35^{\circ}$ or less resulted in posterior cortical breakage in all the knees. 


\section{Discussion}

The principle finding of our research was that the tunnel length was positively correlated with the sagittal inclination and that the tunnel length was longer than $35 \mathrm{~mm}$ in $95 \%$ when the sagittal inclination was between $50^{\circ}$ and $65^{\circ}$ and the axial angle was between $30^{\circ}$ and $45^{\circ}$.

AM portal drilling of the femoral tunnel facilitates creation of a femoral tunnel at native ACL insertion ${ }^{3,14)}$ but results in shorter tunnels ${ }^{6-8)}$ and increases the risk of posterior cortical breach ${ }^{6}$. A recent clinical trial has also found a tendency for shorter tunnels with use of the AM portal technique ${ }^{15}$. In a study of post-operative CT scans following double bundle ACL reconstruction, a femoral tunnel of less than $30 \mathrm{~mm}$ was found to develop in some cases $^{16)}$.

Greis et al. ${ }^{9)}$ reported that the length of a tendon placed within a bone tunnel influences tendon pull-out strength and advocated maximising tendon length inside the bone tunnel. A recent study by Zantop et al. ${ }^{17)}$ did not find any inferior deleterious outcome with insertion of a graft as little as $15 \mathrm{~mm}$ in a goat model. However, graft inset of $20 \mathrm{~mm}$ has been the general recommendation for ACL reconstruction ${ }^{18)}$. Very short femoral tunnels would cause difficulties while using suspensory method of fixation that is considered as the most optimal method ${ }^{18-20)}$. Suspensory fixation devices, such as Tight Rope (Arthrex Inc, Naples, FL, USA), have been attempted as a solution for short tunnels. However even with the use of such devices, short tunnels would result in either a thin cortical bridge or short graft inset length. A thin cortical bridge reduces the margin of error during tunnel drilling in spite of the risk of cortical breakage. Hence, a tunnel length of at least $35 \mathrm{~mm}$ remains a desirable target.

Earlier studies on the length of femoral tunnel in AM portal drilling ${ }^{4,10,11)}$ were cadaveric studies and consequently the number of parameters that can be analysed in a single study was limited and small variations were not reported. These studies have also been limited by lack of standardisation of the entry point of femoral tunnel. A CT-based study would allow an analysis of the influence of hip and knee flexion as well as axial plane orientation of guidewire simultaneously. Additionally, this method is sensitive to minor variations in tunnel orientations.

This study was aimed to estimate the femoral tunnel length using CT measurements. We created virtual tunnels in $3 \mathrm{D}$ reconstructed CT scans and measured the tunnel length in many possible tunnel orientations. The technique of making virtual tunnels on CT scans was devised based on careful analysis of the surgical technique and knowledge of CT measurements. A femoral tunnel is seen as a line in 3D space and such a line is conventionally defined by its end points or its angular relation to the three planes. Though the tunnel entry point on the medial wall of lateral condyle is fixed, its exit on the lateral cortex may vary. Additionally, tunnel orientation in all three planes cannot be measured unless we use computer assisted navigation. Hence, we used a unique method of defining a line in space by defining inclination of the line's projection in one plane (SI) and then defining line by its angular relationship (AA) in the inclined plane along the line's projection (Fig. 3). The orientation of a tunnel in three planes was resolved into two angles that can be measured surgically and reproduced on an analysis of CT. We can measure the sagittal plane projection of the tunnel and the real angle of the tunnel on the inclined plane i.e., the plane parallel to the ground when the knee is fully flexed and guide wire is parallel to the ground (Fig. 4). Whenever our guidewire is not parallel to the ground, we can measure the axial angle along the plane of guidewire.

\section{Sagittal Inclination}

Tunnel orientation in the sagittal plane depends on the hip flexion and the sagittal angle of guidewire insertion and knee flexion influences both the degree of hip flexion and angle of guidewire insertion (Fig. 4). We noted that an increase in sagittal inclination of tunnel resulted in a corresponding increase in tunnel length only at the larger axial angles. At smaller axial angles, increasing the sagittal inclination has little effect on the tunnel length but helps to avoid posterior cortical breakage. This explains the current confusion in the literature regarding the influence of knee flexion on the tunnel length. Basdekis et al. ${ }^{11)}$ noted that increasing knee flexion increases the tunnel length and decreases the risk of posterior cortical breakage. Bedi et al. ${ }^{4)}$ reported that increasing knee flexion reduces the tunnel length. However, the axial angle was not constant in both studies and its confounding effect was not commented. The two studies used cadaveric knees with thigh gripped in a vice. This is different from the position we used in surgery, wherein knee flexion caused hip flexion and thereby contributed to increased sagittal inclination of the tunnel. We recommend creation of femoral tunnel with high sagittal inclination by maximal knee flexion. The analysis of the results of our study reveals that sagittal inclination of more than $50^{\circ}$ results in tunnel length of more than $34 \mathrm{~mm}$ in most axial angles (Table 1, Fig. 7). When tunnels have a high sagittal inclination, they have optimal tunnel length regardless of axial angle and the incidence of posterior cortical breach is less. In contrast, when sagittal inclination is less, tunnel length varies widely with axial angle (Fig. 7). 


\section{Axial Angle}

Hamilton et al reported that the length of femoral tunnel reduces with increased axial angle of drilling ${ }^{10)}$. Their study was done with the knee in $120^{\circ}$ flexion. We also noted that the tunnel length is longer at a smaller axial angle. Moreover, at smaller axial angles, the tunnel is consistent with various sagittal inclinations. However, the incidence of posterior cortical breakage was greater at smaller axial angles especially when sagittal inclination was also low. A combination of low sagittal and low axial angles results in posterior cortical breakage, while low sagittal and high axial angles result in very short tunnels. From our study, we can conclude that drilling the femoral tunnel with the knee maximally flexed and guidwire set at an axial angle of about $30^{\circ}-45^{\circ}$ is the reliable method to safely achieve optimal tunnel length. This tunnel orientation results in an optimal tunnel length of 35-45 mm and minor errors in tunnel orientation will have less effect on the tunnel length because when the knee is maximally flexed and sagittal inclination of tunnel is about $60^{\circ}-65^{\circ}$, the tunnel length is consistent. The results of our study concur with the study on the femoral tunnel length by Tompkins et al. ${ }^{21)}$. The authors employed maximal knee flexion while drilling the femoral tunnel through an AM portal and reported an average tunnel length of $37 \mathrm{~mm}^{21)}$.

Complex morphology of distal femur dictates that the tunnel orientation has a critical influence on the length of femoral tunnel. A $<40^{\circ}$ of sagittal inclination was associated with wide variation of tunnel length and variations in axial angles and sagittal inclination affected the tunnel length only when axial angle was greater than $40^{\circ}$. Further studies on the relationship between the entry point and lateral cortex of the femur are needed to investigate this phenomenon. At a higher sagittal inclination, the tunnel length was longer regardless of the change in the axial angle. Hence, the surgeon should strive to increase the sagittal inclination and maintain the axial angle at $30^{\circ}-45^{\circ}$.

Our study has some limitations, this is a CT study based on observations during surgery and hence the influences of portal positioning and soft tissues on the tunnel orientation were not assessed. The tunnel lengths were measured by drawing virtual tunnels on CT images. The surgeon who would follow our recommendations must be aware of the potential difficulties that might be encountered. Achieving accurate tunnel orientation during surgery requires accurate measurement of angles with a goniometer. Portal placement must be meticulous to avoid interference during surgery. Two angles that must be considered are the sagittal inclination, which must be measured only on the sagittal plane and the axial angle that must be measured in the plane of guidewire, not just in the plane parallel to floor (Fig. 3). Care must be taken to identify the true sagittal line to measure the axial angle from. Extremely low axial angles might result in posterior slipping of drill and consequent posterior drilling. Moreover, maximal knee flexion would cause difficulties in visualisation of the notch. Use of a central medial visualisation portal or a $70^{\circ}$ scope has been suggested by other authors to obviate this difficulty.

\section{Conclusions}

A tunnel length of more than $35 \mathrm{~mm}$ can be created by achieving a sagittal inclination of greater than $45^{\circ}$ and an axial angle of $30^{\circ}-45^{\circ}$. Such an orientation can be obtained by flexing the knee fully so that the hip flexion increases so as to increase the sagittal inclination.

\section{Conflict of Interest}

No potential conflict of interest relevant to this article was reported.

\section{References}

1. Asano H, Muneta T, Ikeda H, Yagishita K, Kurihara Y, Sekiya I. Arthroscopic evaluation of the articular cartilage after anterior cruciate ligament reconstruction: a short-term prospective study of 105 patients. Arthroscopy. 2004;20:474-81.

2. Pastrone A, Ferro A, Bruzzone M, Bonasia DE, Pellegrino P, D'Elicio D, Cottino U, Rossi R. Anterior cruciate ligament reconstruction creating the femoral tunnel through the anteromedial portal. Surgical technique. Curr Rev Musculoskelet Med. 2011;4:52-6.

3. Tompkins M, Milewski MD, Brockmeier SF, Gaskin CM, Hart JM, Miller MD. Anatomic femoral tunnel drilling in anterior cruciate ligament reconstruction: use of an accessory medial portal versus traditional transtibial drilling. Am J Sports Med. 2012;40:1313-21.

4. Bedi A, Raphael B, Maderazo A, Pavlov H, Williams RJ 3rd. Transtibial versus anteromedial portal drilling for anterior cruciate ligament reconstruction: a cadaveric study of femoral tunnel length and obliquity. Arthroscopy. 2010;26:34250.

5. Kim MK, Lee BC, Park JH. Anatomic single bundle anterior cruciate ligament reconstruction by the two anteromedial portal method: the comparison of transportal and transtibial 
techniques. Knee Surg Relat Res. 2011;23:213-9.

6. Chang CB, Choi JY, Koh IJ, Lee KJ, Lee KH, Kim TK. Comparisons of femoral tunnel position and length in anterior cruciate ligament reconstruction: modified transtibial versus anteromedial portal techniques. Arthroscopy. 2011;27:138994.

7. Lubowitz JH, Konicek J. Anterior cruciate ligament femoral tunnel length: cadaveric analysis comparing anteromedial portal versus outside-in technique. Arthroscopy. 2010;26:1357-62.

8. Miller CD, Gerdeman AC, Hart JM, Bennett CG, Golish SR, Gaskin C, Miller MD. A comparison of 2 drilling techniques on the femoral tunnel for anterior cruciate ligament reconstruction. Arthroscopy. 2011;27:372-9.

9. Greis PE, Burks RT, Bachus K, Luker MG. The influence of tendon length and fit on the strength of a tendon-bone tunnel complex. A biomechanical and histologic study in the dog. Am J Sports Med. 2001;29:493-7.

10. Hamilton SC, Jackson ER 2nd, Karas SG. Anterior cruciate ligament femoral tunnel drilling through anteromedial portal: axial plane drill angle affects tunnel length. Arthroscopy. 2011;27:522-5.

11. Basdekis G, Abisafi C, Christel P. Influence of knee flexion angle on femoral tunnel characteristics when drilled through the anteromedial portal during anterior cruciate ligament reconstruction. Arthroscopy. 2008;24:459-64.

12. Bird JH, Carmont MR, Dhillon M, Smith N, Brown C, Thompson P, Spalding T. Validation of a new technique to determine midbundle femoral tunnel position in anterior cruciate ligament reconstruction using 3-dimensional computed tomography analysis. Arthroscopy. 2011;27:1259-67.

13. Taro Y. Statistics: an introductory analysis. 3rd ed. New York: Haper \& Row; 1973. p205.
14. Bedi A, Musahl V, Steuber V, Kendoff D, Choi D, Allen AA, Pearle AD, Altchek DW. Transtibial versus anteromedial portal reaming in anterior cruciate ligament reconstruction: an anatomic and biomechanical evaluation of surgical technique. Arthroscopy. 2011;27:380-90.

15. Ilahi OA, Ventura NJ, Qadeer AA. Femoral tunnel length: accessory anteromedial portal drilling versus transtibial drilling. Arthroscopy. 2012;28:486-91.

16. Wang JH, Kim JG, Ahn JH, Lim HC, Hoshino Y, Fu FH. Is femoral tunnel length correlated with the intercondylar notch and femoral condyle geometry after double-bundle anterior cruciate ligament reconstruction using the transportal technique? An in vivo computed tomography analysis. Arthroscopy. 2012;28:1094-103.

17. Zantop T, Ferretti M, Bell KM, Brucker PU, Gilbertson L, Fu FH. Effect of tunnel-graft length on the biomechanics of anterior cruciate ligament-reconstructed knees: intra-articular study in a goat model. Am J Sports Med. 2008;36:2158-66.

18. Strobell MJ. Manual of arthroscopic surgery. 1st ed. Berlin: Springer-Verlag; 2002. p460.

19. Hoher J, Livesay GA, Ma CB, Withrow JD, Fu FH, Woo SL. Hamstring graft motion in the femoral bone tunnel when using titanium button/polyester tape fixation. Knee Surg Sports Traumatol Arthrosc. 1999;7:215-9.

20. Milano G, Mulas PD, Ziranu F, Piras S, Manunta A, Fabbriciani C. Comparison between different femoral fixation devices for ACL reconstruction with doubled hamstring tendon graft: a biomechanical analysis. Arthroscopy. 2006;22:660-8.

21. Tompkins M, Milewski MD, Carson EW, Brockmeier SF, Hamann JC, Hart JM, Miller MD. Femoral tunnel length in primary anterior cruciate ligament reconstruction using an accessory medial portal. Arthroscopy. 2013;29:238-43. 